\title{
Single-Cell Tumor Phylogeny Inference with Copy-Number Constrained Mutation Losses
}

\author{
Gryte Satas $^{1,2}$, Simone Zaccaria ${ }^{1}$, Geoffrey Mon ${ }^{1}$, \\ and Benjamin J. Raphael ${ }^{1(\otimes)}$ \\ 1 Department of Computer Science, Princeton University, Princeton, NJ 08544, USA \\ braphael@princeton.edu \\ 2 Department of Computer Science, Brown University, Providence, RI 02906, USA
}

Motivation: Single-cell DNA sequencing enables the measurement of somatic mutations in individual tumor cells, and provides data to reconstruct the evolutionary history of the tumor. Nearly all existing methods to construct phylogenetic trees from single-cell sequencing data use single-nucleotide variants (SNVs) as markers. However, most solid tumors contain copy-number aberrations (CNAs) which can overlap loci containing SNVs. Particularly problematic are CNAs that delete an SNV, thus returning the SNV locus to the unmutated state. Such mutation losses are allowed in some models of SNV evolution, but these models are generally too permissive, allowing mutation losses without evidence of a CNA overlapping the locus.

Results: We introduce a novel loss-supported evolutionary model, a generalization of the infinite sites and Dollo models, that constrains mutation losses to loci with evidence of a decrease in copy number. We design a new algorithm, SCAR-

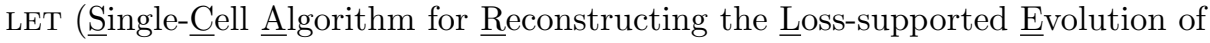
Tumors), that infers phylogenies from single-cell tumor sequencing data using the loss-supported model and a probabilistic model of sequencing errors and allele dropout. On simulated data, we show that SCARLET outperforms current single-cell phylogeny methods, recovering more accurate trees and correcting errors in SNV data. On single-cell sequencing data from a metastatic colorectal cancer patient, SCARLET constructs a phylogeny that is both more consistent with the observed copy-number data and also reveals a simpler monoclonal seeding of the metastasis, contrasting with published reports of polyclonal seeding in this patient. SCARLET substantially improves single-cell phylogeny inference in tumors with CNAs, yielding new insights into the analysis of tumor evolution.

Availability: Software is available at github.com/raphael-group/scarlet Preprint: A preprint of the manuscript is available at https://www.biorxiv.org/content/10.1101/840355v1 